\title{
DETECTING BRAIN TUMOUR FROM MRI IMAGE USiNg Matlab GUI ProgRamme
}

\author{
Esmail Hassan $^{1}$ and Abobakr Aboshgifa ${ }^{2}$ \\ ${ }^{1}$ Higher Institute of Medical Professions - Tripoli Libya \\ ${ }^{2}$ High technical center for training and production. Tripoli Libya.
}

\begin{abstract}
Engineers have been actively developing tools to detect tumors and to process medical images. Medical image segmentation is a powerful tool that is often used to detect tumors. Many scientists and researchers are working to develop and add more features to this tool. This project is about detecting Brain tumors from MRI images using an interface of GUI in Matlab. Using the GUI, this program can use various combinations of segmentation, filters, and other image processing algorithms to achieve the best results. We start with filtering the image using Prewitt horizontal edge-emphasizing filter. The next step for detecting tumor is "watershed pixels." The most important part of this project is that all the Matlab programs work with GUI "Matlab guide". This allows us to use various combinations of filters, and other image processing techniques to arrive at the best result that can help us detect brain tumors in their early stages.
\end{abstract}

\section{KEYWORDS}

Matlab program, GUI program, Tumors, Medical image.

\section{INTRODUCTION}

In these days, the interest in digital biomedical image processing methods takes a most important position in two principal and important areas[1]. The most important one is an improvement of pictorial information for human studies and processing of biomedical image data for storage. A biomedical image sometimes is defined as a two-dimensional function, $\mathrm{F}(\mathrm{x}, \mathrm{y})$, where $\mathrm{x}$ and $\mathrm{y}$ are the value or gray level of a biomedical image at a specific point. $F$ are all finite, discrete quantities. We should know that to say an image is a digital image is when it is composed of a finite number of elements, each of which has a particular location and value. For example figure (1) shows the setting date for an MRI image with a gray level according to researchers at the University at Buffalo School of Medicine and Biomedical Sciences. They have reported the MRI results of patients who were diagnosed with multiple sclerosis in childhood. They take MRI images during the year to diagnose and study the progress of brain disease [2].

Biomedical images are as different as the areas of the human body. For example, to study soft tissue in the human body, we have to use the MRI scan for soft tissue images such as Brain liver and other soft tissue in the human body. However, those interested in studying hard tissue such as bone or cartilage should use X-ray for a hard tissue image rather than the MRI. The difference in the biomedical image is not just in the area but also different in the manner of processing. In 
other words, to process an MRI image it is necessary to use a different method than for processing an X-ray image.

$$
I_{\text {input }}--T--\rightarrow I_{\text {output }}=T\left[I_{\text {input }}\right]
$$

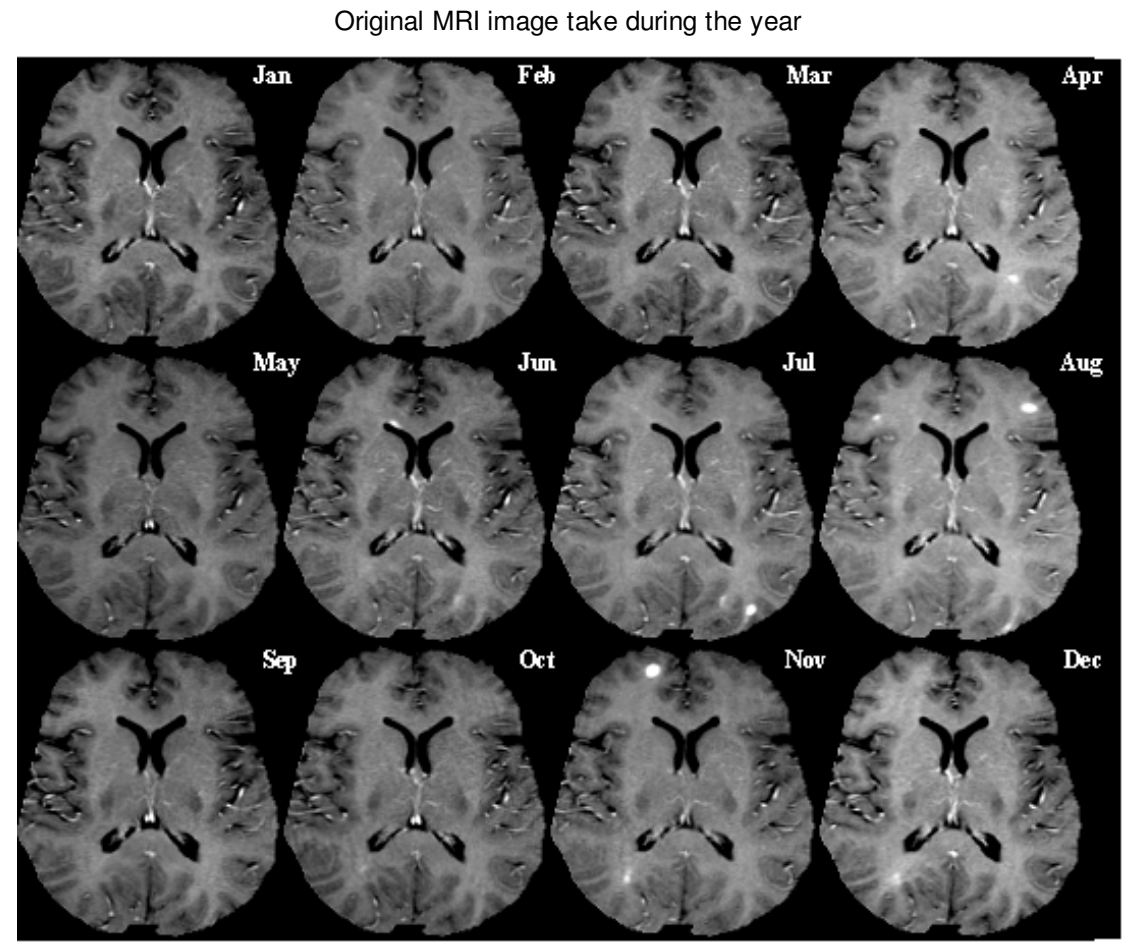

Figure 1 .showing one example of studying the brain during the year using MRI images.

\section{Specific Problem With MRI Image Segmentation}

As all the development of biomedical image processing has gained attention from the scientists, there are some problems with biomedical image processing that have appeared, particularly with MRI imaging. The one general problem with the biomedical image segmentation (MRI image) method for image segmentation is that it varies widely depending on the specific application [2]. For example, the segmentation program that is used with MRI imaging has different requirements from segmentation of CT scan imaging. Furthermore, each image, even if it is from the same image application, such as MRI, has its own idiosyncrasies. These can be different from other MRI images, which when read by the same segmentation program will give a different result. In fact, there is no segmentation method that yields acceptable results for every medical image that are more general and can be applied to a variety of data. However, segmentation methods that are specialized to one imaging application can often achieve better results by taking into account prior knowledge [3]. The image processing segmentation which applies to Magnetic resonance images (MRI) are complicated due to the limitation of the imaging equipment.

There is a further problem with segmentation in the MIR image which exists in the homogeneous aspects of pixels in imaging regions that make getting information about the image's subject 
difficult because the MRI image is all about soft tissue such as brain tissue or liver tissue. Homogeneous aspects of pixels in the MRI image make the segmentation program unsuccessful with all the MRI image, so the result for one image might be different from another image. In this case, we have to change the setting information for the segmentation program [12].

\section{Some Ideas for MRI Image Processing}

Detecting brain tumor and automatic brain tissue classification from magnetic resonance images (MRI) is very important for research and clinical studies of the normal and diseased human brain [14]. The most important method used to processes an MRI image is segmentation of image which works by dividing the objects in the image and processing them separately. There are three important segmentation methods used with MRI imaging:

1. Classification based

2.Region based

3. Contour based

The complexity and reality of anatomical information presented by MRI have made it a powerful tool for medical diagnosis in recent years. However, usually, the researcher is looking for a better result so he tries to build and create a more powerful tool that can give a clear idea of an MRI image and also to aid in the study and diagnosis of disease. One of those powerful tools used with MRI image processing is image segmentation [15]. Image segmentation can be used in different ways and can provide different results. For example the way of using region growing segmentation is different from watershed segmentation; even the result is different. However, using segmentation programs sometimes is complicated because it takes the time to process the image and it is difficult to use the same program with all images. The Manual segmentation program has also some problems with MRI image making the segmentation impossible to reproduce and/or deteriorating the result of the segmentation. Hence, there is a real need for automated MRI segmentation tools which save time and provide better and more consistent results [15].

\section{Combine Mri Image Processing Methods in one Place}

This study is about comparing MRI image processing methods with detecting the brain tumor from MRI image. Start researching the idea to have the methods in one place showing their results at the same time [19]. If the GUI program is easy to control, add segmentation and other MRI image processing methods as function tool to the GUI "guide" as shown in figure (12). 
International Journal of Computer Science \& Engineering Survey (IJCSES) Vol.6, No.6, December 2015

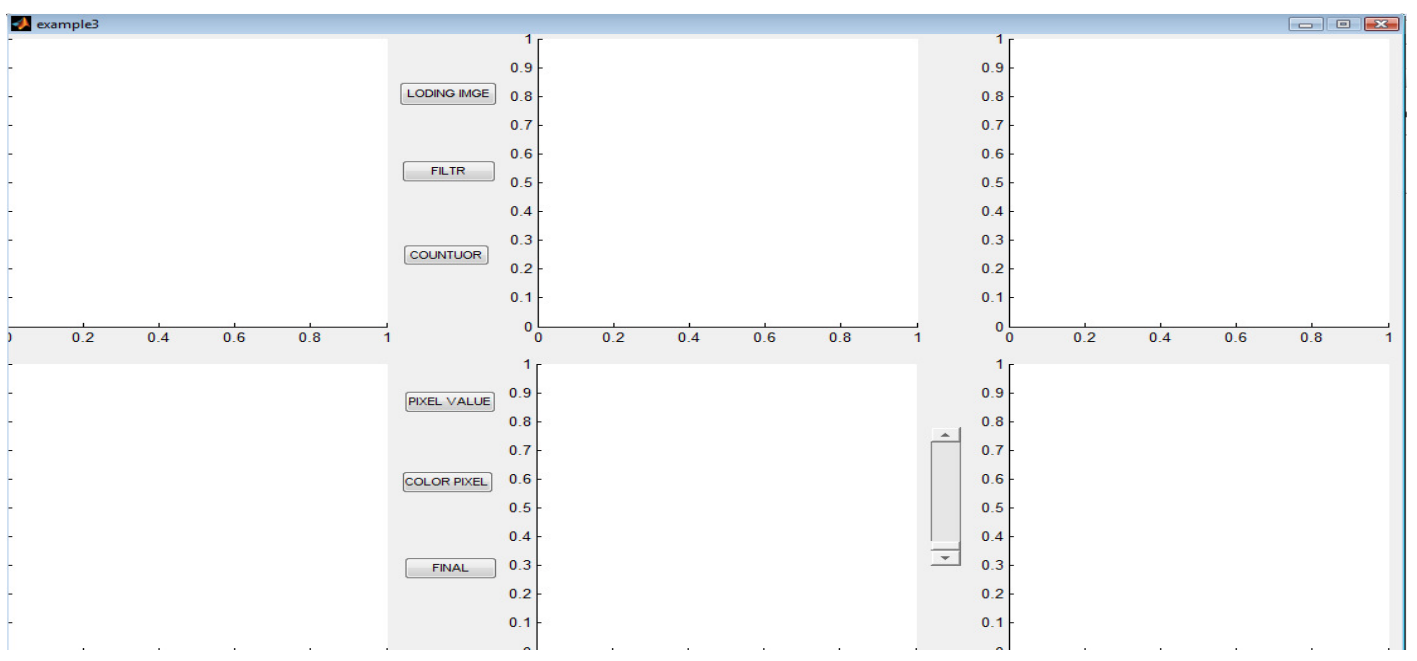

Figure15. interface for GUI to control MRI image processing

As can be seen in the figure, there are six push buttons. Each button has a function as part of the program and also there are six axes showing the resulting image starting with loading the original image of the MRI [18]. With help from GUI, we can have a program that runs fast and provides a better result with more methods.

\section{DeteCting BRaIN TUMOR USING IMAge SEgMENTATION WITH GUI "MATLAB GUIDE".}

The first thing to know is what is the GUIDE program "GUI"? It is the Matlab interface File used to hold and process information, a function for GUI FIG-files created or modified using MATLAB. The GUI is a modern tool that can be found with 0.7 versions or a later MATLAB version not automatically compatible with Version 6.5 and earlier versions[19]. GUIDE automatically generates two kinds of Matlab files; one is for Matlab interface figures and another is for M-file, used to store the command function of the Matlab program [16]. Before, a Matlab user had to write the Matlab logarithm in an M-file and if there were any changes they had to go back to the M-file to make the change. But now, using GUI we can use the logarithm as a function in the GUI file [12]. Writing the function that we need the GUI Matlab file to do: first, we should organize the most important part. There are many ways to start GUIDE. You can start GUIDE from the Command line by typing guide or start menu by selecting MATLAB > GUIDE (GUI Builder). However you start GUIDE, it displays the GUIDE Quick Start dialog box as shown in figure(13). 


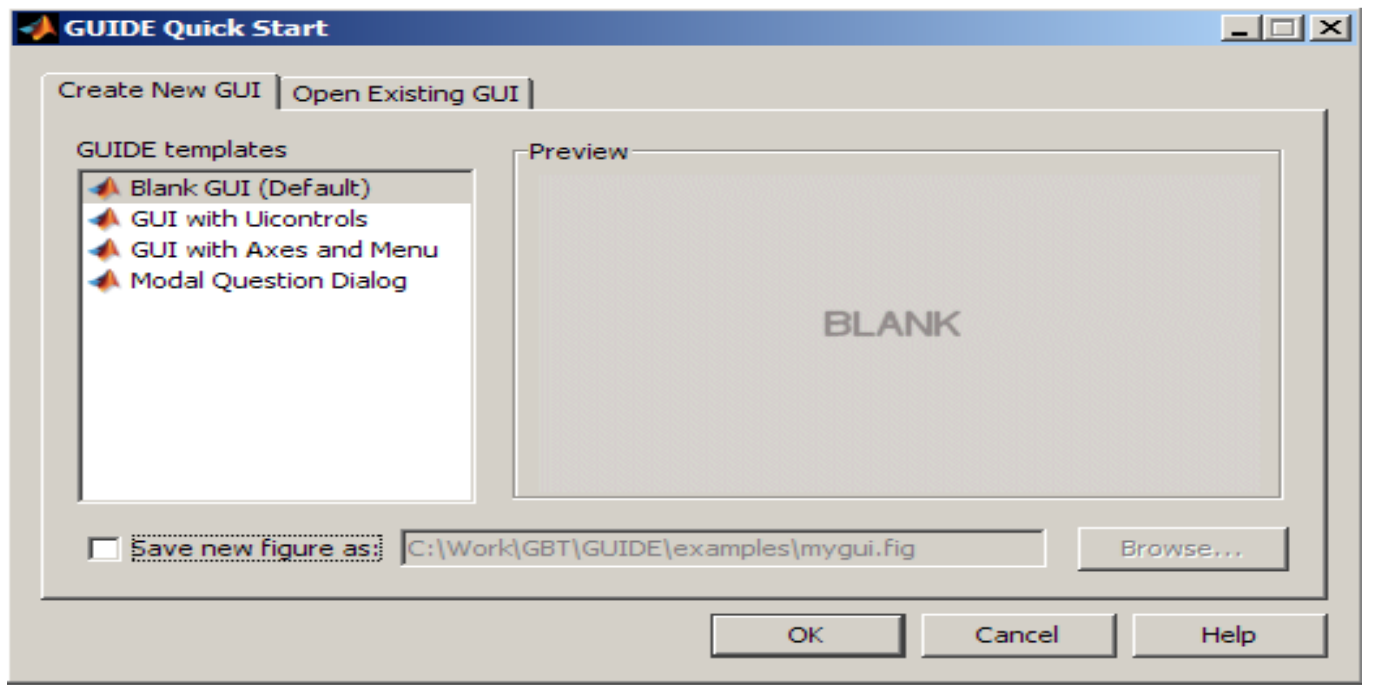

Figure16. Showing GUI blank function file

By using the GUI Layout Editor, you can populate a GUI by clicking and dragging GUI components - such as axes, panels, buttons, text fields, sliders, and so on-into the layout area. You can also create menus and context menus for the GUI [19]. From the Layout Editor, you can change the size of GUI interface. How the GUI program working GUIDE automatically generates an M-file that controls how the GUI operates. This M-file provides code to initialize the GUI and contains a framework for the GUI click-backs, the routines that execute when a user interacts with a GUI component. Using the M-file editor, you can add code to the click-backs to perform the functions you want [18]. After we site the GUI interface function as in figure (14), we have to write the click-back function that will be the tumor segmentation program.

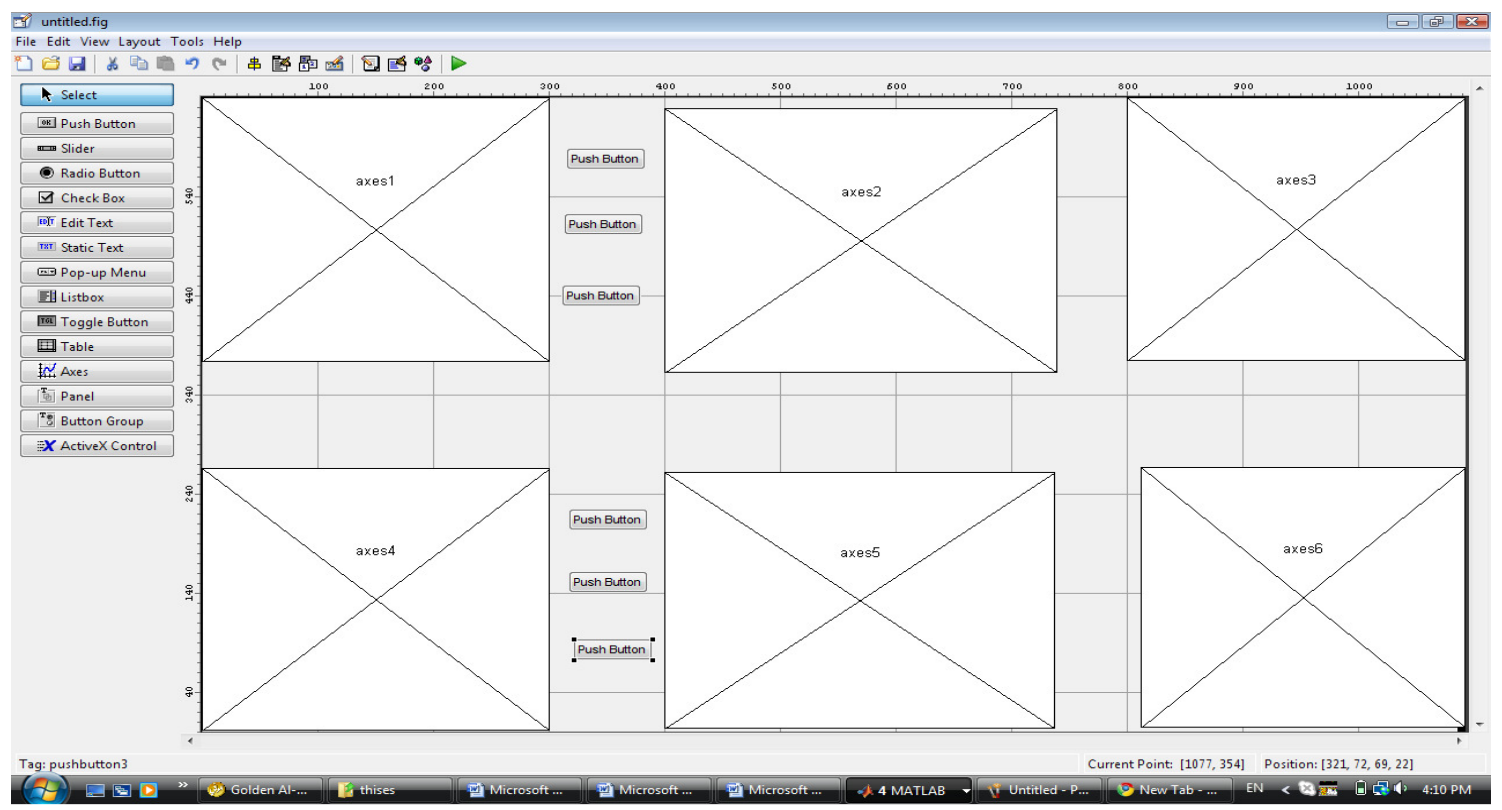

Figure 17. Showing the GUI interface for MRI segmentation program. 


\section{METHODS OF DETECTING BRAIN TUMOR FROM MRI.}

Methods that we use to detect brain tumor from MRI images (figure 15) are watershed segmentation and contour of the image [17]. Before we start the segmentation we have to filter the MRI image noise. We use in this step the digital filter from Matlab ("Sobel edge masks") which will show the gradient is high at the borders of the MRI objects and low (mostly) inside the image as shown in figure(16).

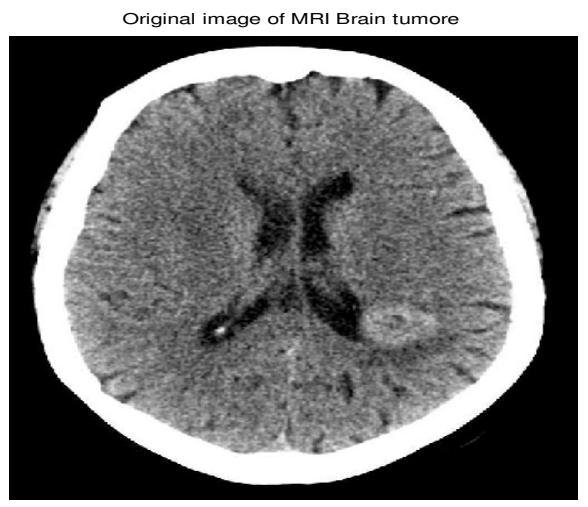

Figure 18. Original MRI images for brain tumor

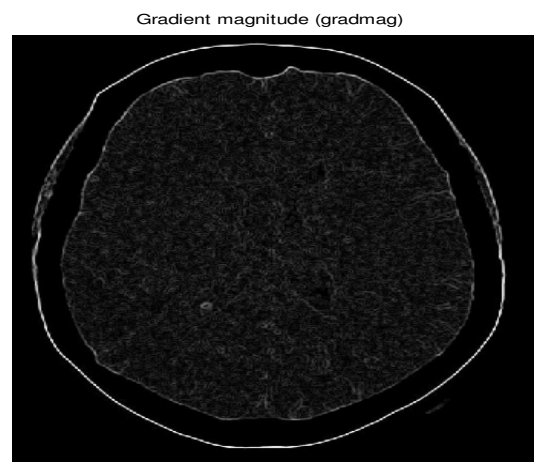

Figure 19. Showing MRI image after using of "Sobel" edge mask

Next step is using the contour program to see the contour of the objects in the MRI image As can be seen in figure (20) 
International Journal of Computer Science \& Engineering Survey (IJCSES) Vol.6, No.6, December 2015

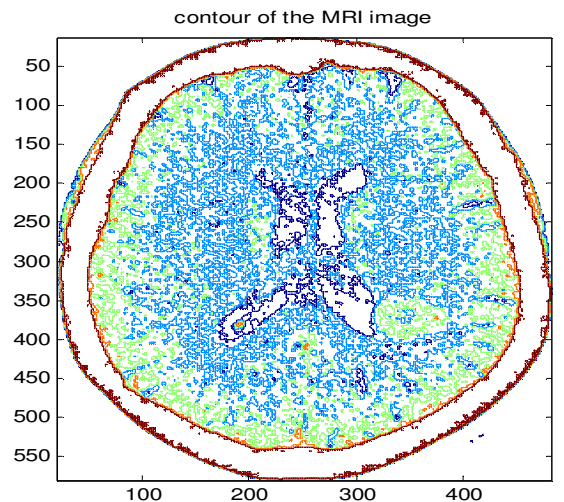

Figure 20. Contour of the MRI image

After we have filtered the noise of the MRI image the next step is finding the gradient magnitude in this step we will find the different in the pixels of the image as shown in figure (21)

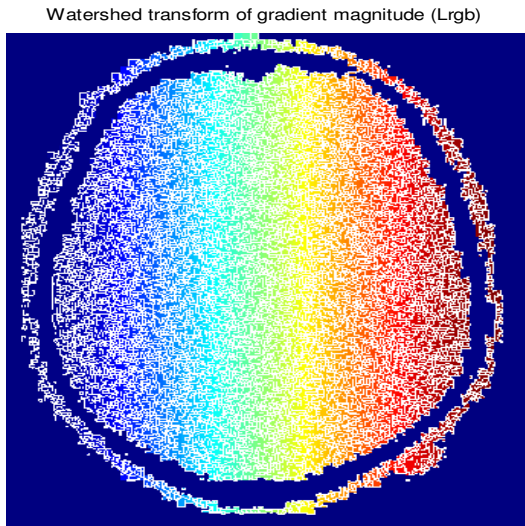

Figure 21. Gradient magnitude of MRI image

Compute the Watershed Transform of the Segmentation Function as clear segmentation for the MRI image. We use the watershed method starting with the transform of gradient Magnitude as can be seen in figure(22) Now we have to make the different pixels as in different region in the MRI image in this way we can detect the region of the tumor as it can be seen in figure (23). 
International Journal of Computer Science \& Engineering Survey (IJCSES) Vol.6, No.6, December 2015

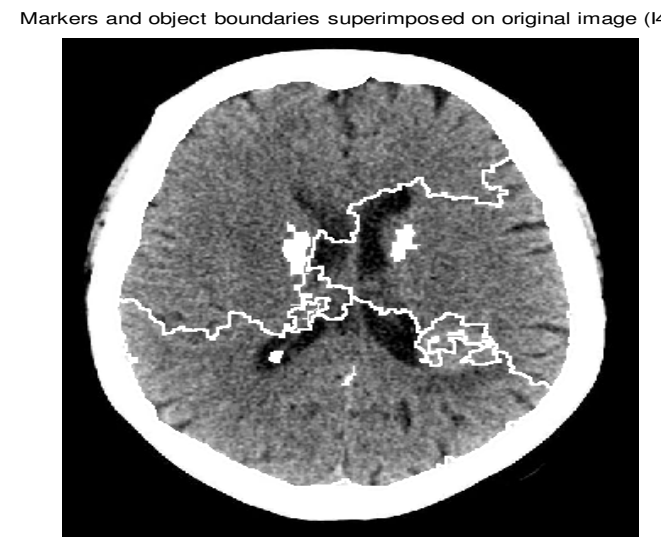

Figure 22. MRI image region's using watershed segmentation

\section{ThE ReSUlts BEFORE USING GUI PROGRAM}

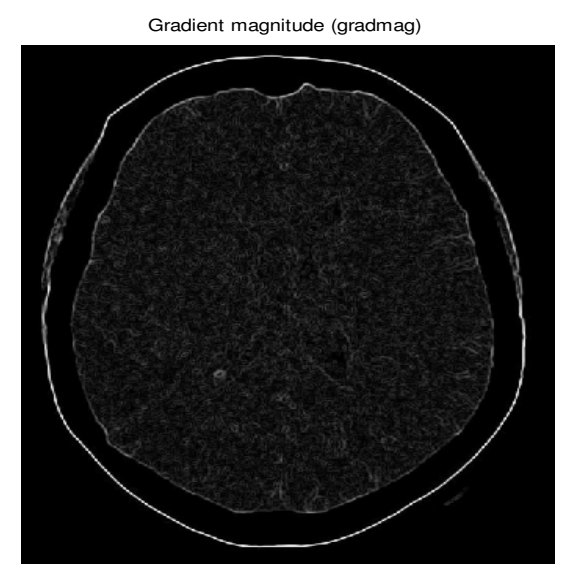

Figure 23. Original Mri Images for Brain Tumor 
International Journal of Computer Science \& Engineering Survey (IJCSES) Vol.6, No.6, December 2015

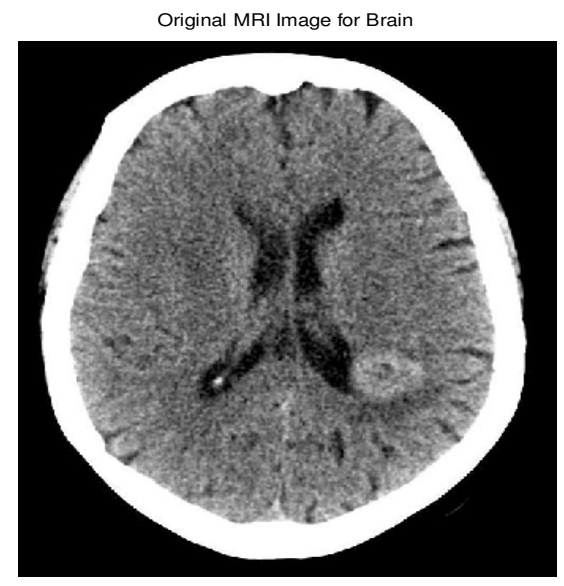

Figure 24. MRI image using Soble Makes

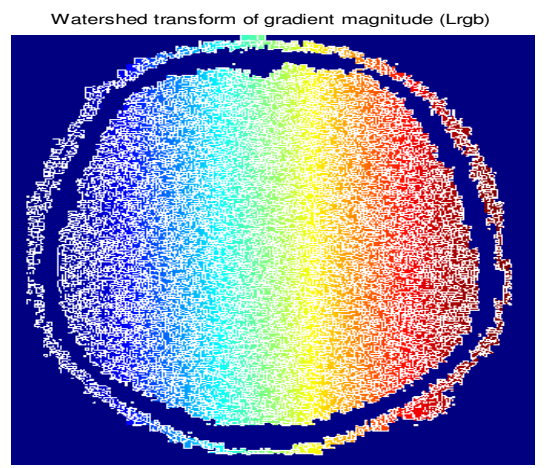

Figure 25. Watershed transform of gradient magnitude ( $\mathrm{Lrgb})$

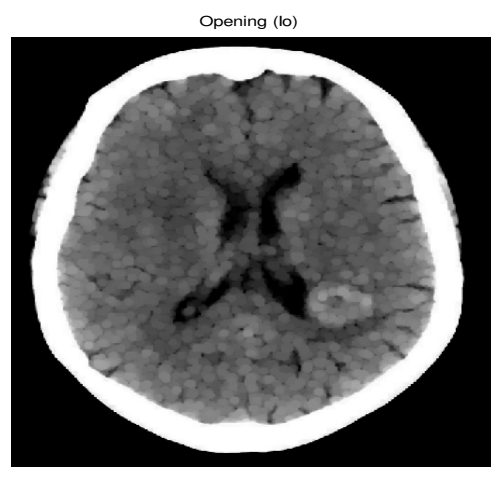

Figure 26. Opening for faltering 
International Journal of Computer Science \& Engineering Survey (IJCSES) Vol.6, No.6, December 2015

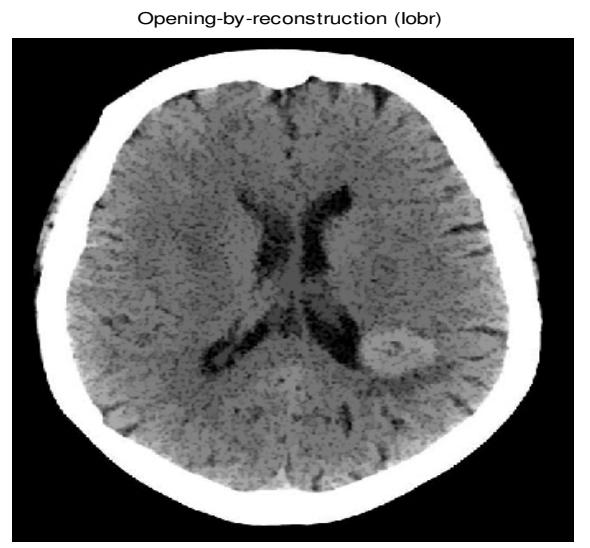

Figure 27. Opening by reconstruction (lobr)

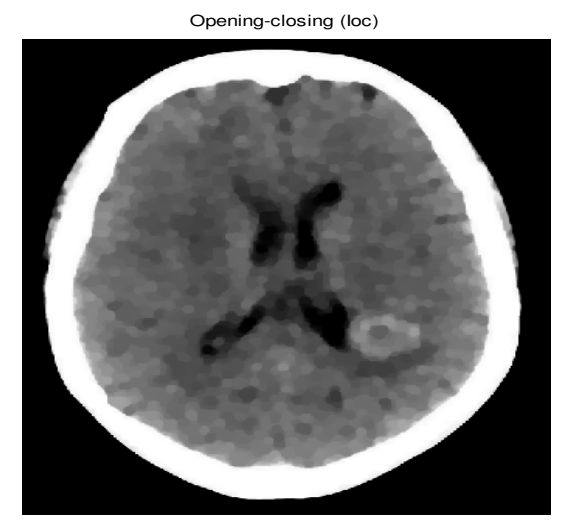

Figure 28. Using Matlab function of (loc)

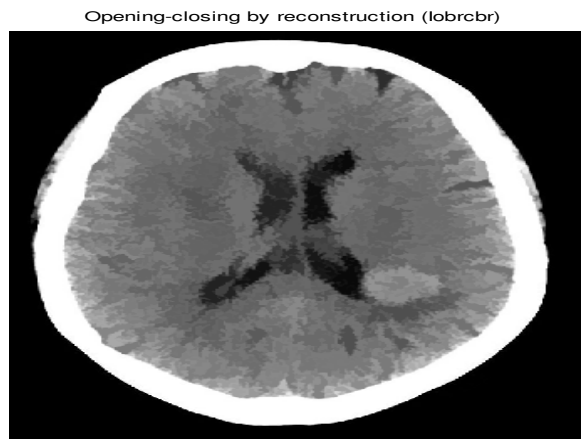

Figure 29. using Matlab function of (lobrcbr) 
International Journal of Computer Science \& Engineering Survey (IJCSES) Vol.6, No.6, December 2015

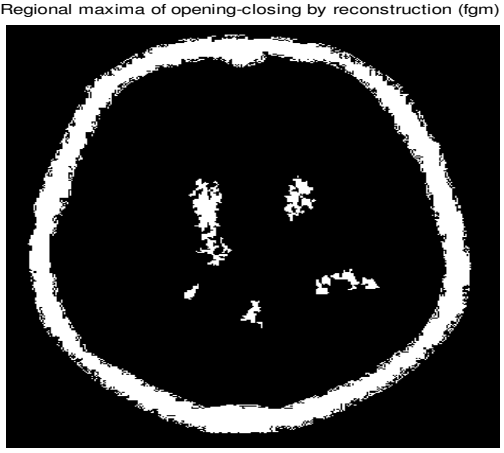

Figure 30. Using reconstruction (fgm)

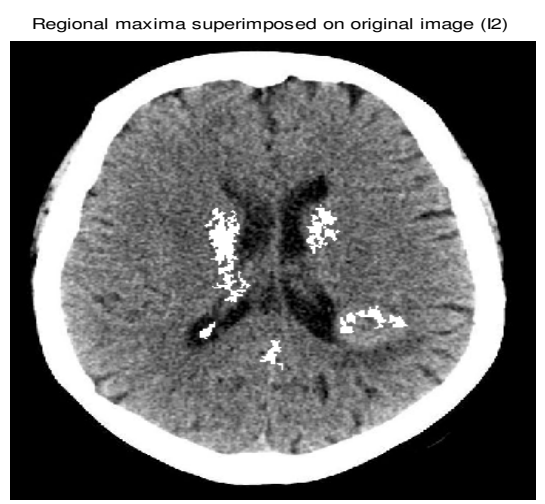

Figure 31. using of superimposed on original MRI image

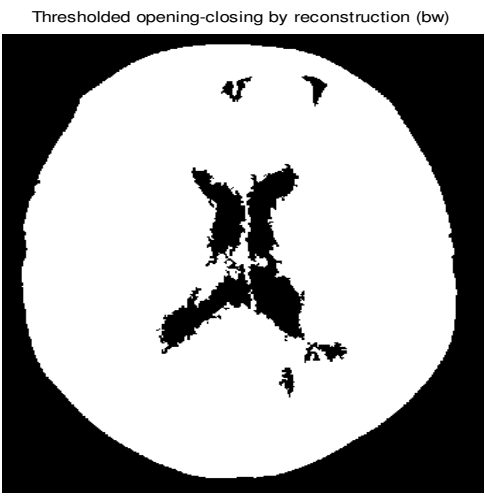

Figure 32. using thresholded 
International Journal of Computer Science \& Engineering Survey (IJCSES) Vol.6, No.6, December 2015

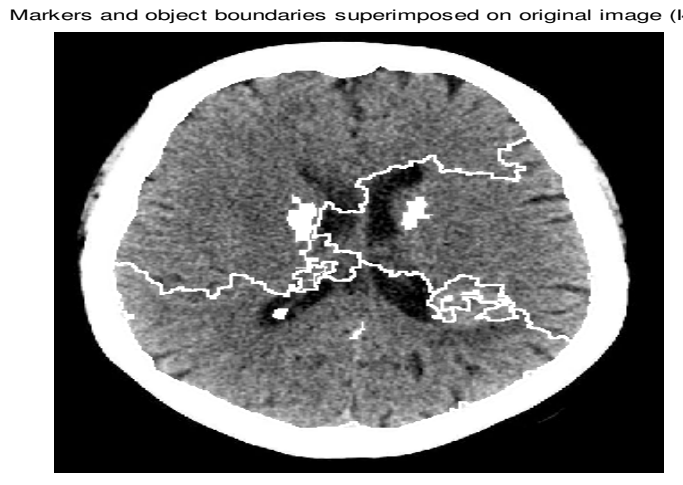

Figure 33. Boundaries of MRI regions

\section{The Final Result Using The GUI Program}

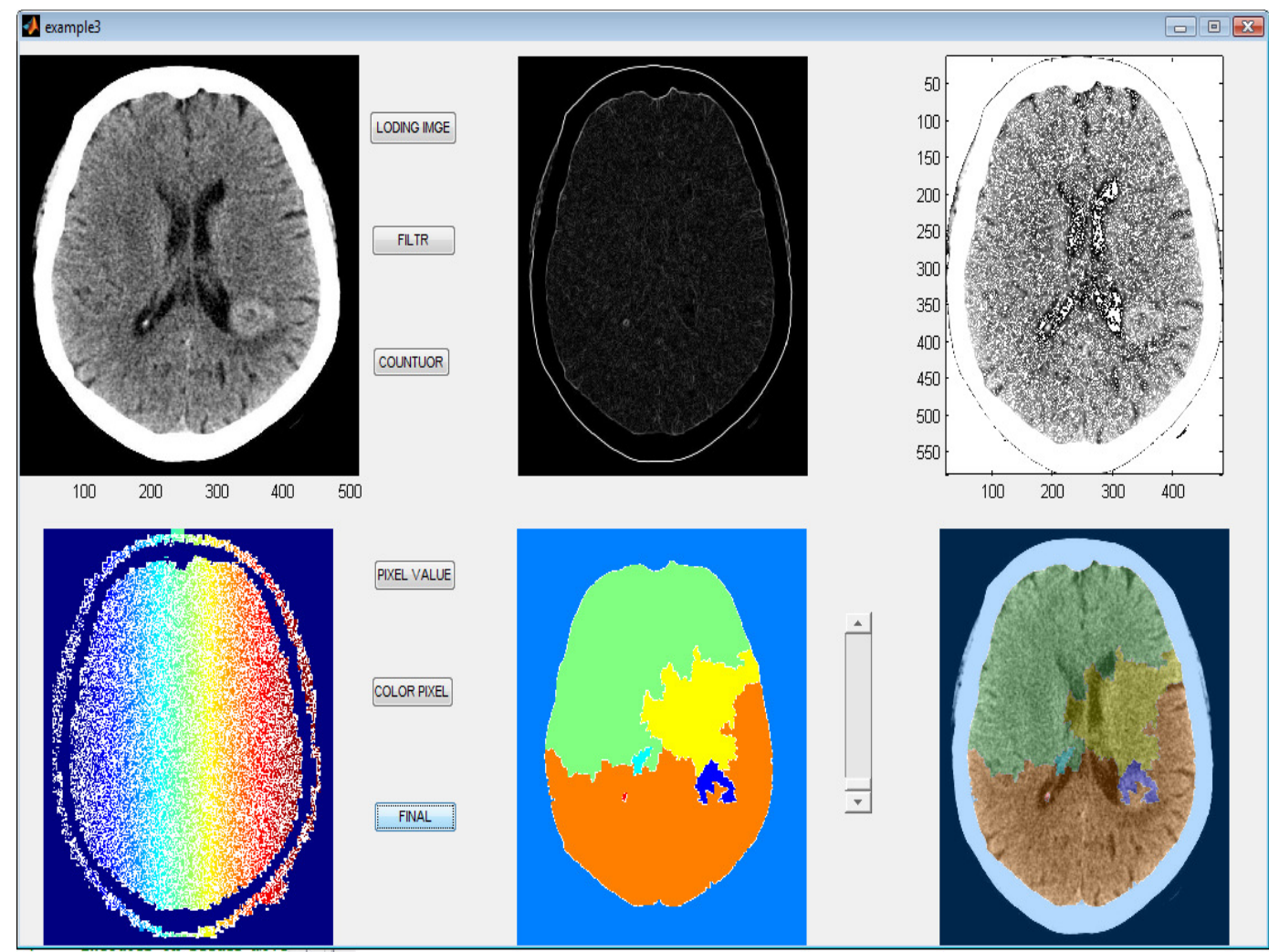

Figure 34. Result after using of GUI with watershed segmentation and contour of MRI image 


\section{To gIVE CleAR IDEA ABOUT PROGRAM WE USE DIFFERENT MRI IMAGES}

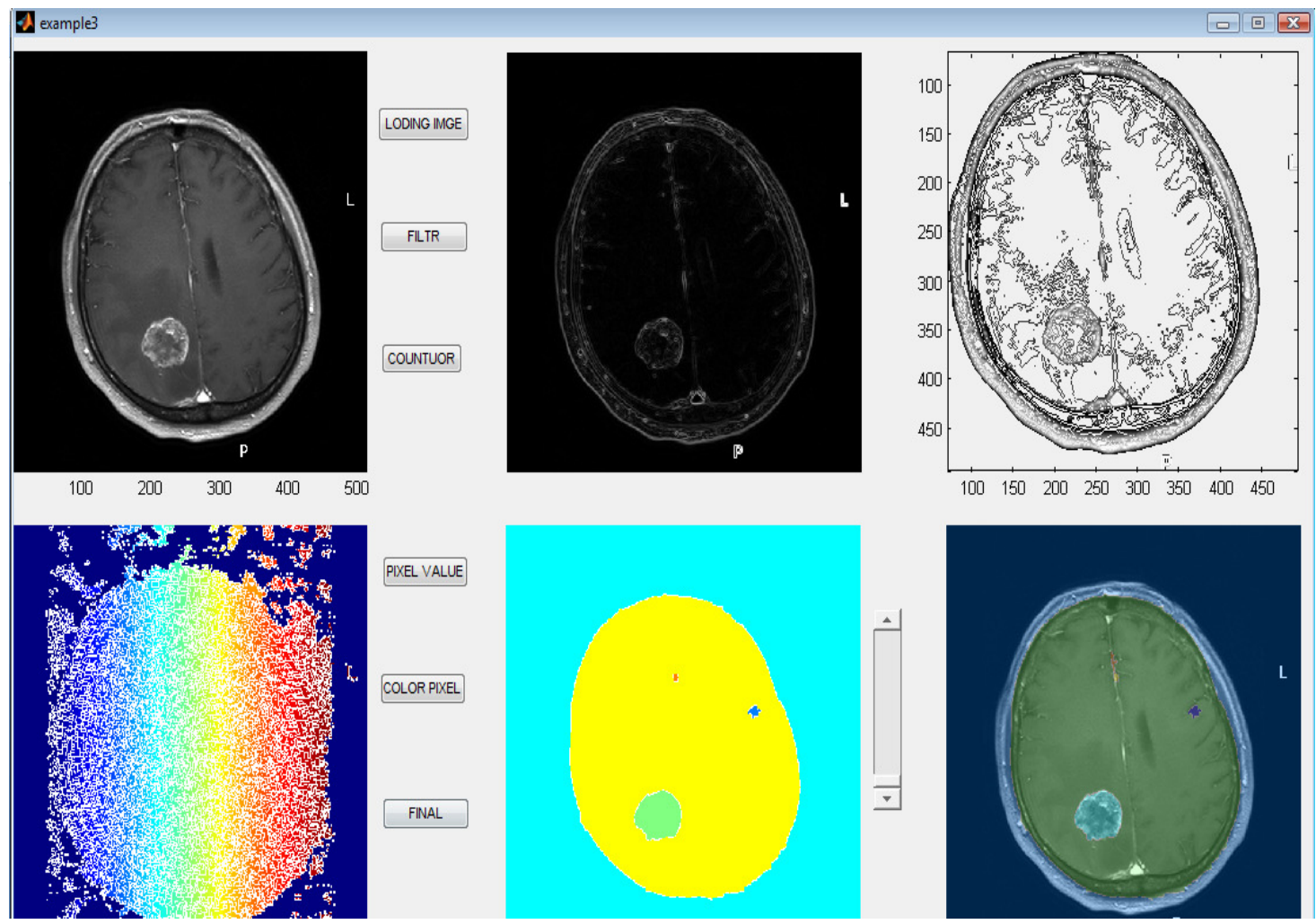

Figure 35. the result after using GUI with another MRI image with a clear Brain tumor

\section{ConClusion}

We see that using the GUI based program, we obtain far superior results than the traditional techniques for tumor detection. Using the GUI based programs allows us to change the parameters without rewriting the program and allows fast and efficient detection of tumors. The results are clearly more accurate and faster.

\section{REFERENCES}

[1] Xia, Y., Bettinger, K., Shen, L.,Reiss, A.: Automatic segmentation of the caudate nucleus from human brain MR images. IEEE Transactions on Medical Imaging 26(4) (2007) 509-517

[2] C.Chu and K. Takaya, "3-Dimensional rendering of MR images," WESCANEX 93. Communications, Computers and Power in the Modern Environment Conference Proceedings, IEEE, pp. 165-170, 2003

[3] Clarke, L., Velthuizen, R., Camacho, M., Heine, J., Vaydianathan, M., Hall, L., Thatcher, R., Silbiger, M.: MRI segmentation: Methods and applications. Magnetic Resonance Imaging 13 (2002)

[4] Cline, H., Lorensen, W., Kikinis, R. and Jolesz, F. (2001) Three- Dimensional Segmentation of MR Images of the Head Using Probability and Connectivity. J. Comp. Assis. Tomogr., 14, 1037-1045 
[5] J.Liang, McInerney, D. Terzopolos Interactive Medical Image Segmentation with United Snakes Medical Image Computing and Computer-Assisted Intervention - MICCAI'99, 116, 1999.

[6] Pham, D.L., Xu, C., Prince, J.L.: Current methods in medical image segmentation. Annual Review of Biomedical Engineering 2 (2000) $315\{337$.

[7] Dugas-Phocion, G., Ballester, M.A.G., Malandain, G., Lebrun, C., Ayache, N.: Improved em-based tissue segmentation and partial volume e®ect quantification In multi-sequence brain MRI. In: MICCAI. (2004)

[8] Kapur, T., Grimson, W.E., Wells, W.M., Kikinis, R.: Segmentation of brain tissue from magnetic resonance images. Med Image Anal. 1 (1996)

[9] S.Haulon, C. Lions, E. McFadden, M. Koussa, V. Gavotte, P. Halna, and J. Beregi, "Prospective evaluation of magnetic resonance imaging after endovascular treatment of infrarenal aortic aneurysms," European Journal of Vascular and Endovascular Surgery 22(1), pp. 62-69, 2001.

[10] Prastawa, M., Gilmore, J., Lin, W., Gerig, G.: Automatic segmentation of Neonatal brain MRI. In: MICCAI. (2004)

[11] B.van Ginneken, M. de Bruijne, M. Loog, and M. Viergever, "Interactive shape models," in Medical Imaging: Image Processing, Proc of SPIE 5032, pp. 1206-1216, SPIE Press, 2003.

[12] Leemput, K.V., Maes, F., Vandermeulen, D., Suetens, P.: Automated model- Based tissue classi $^{-}$cation of MR images of the brain. IEEE trans. on medical imaging 18 (1999) 897

[13] Zhang, Y., Brady, M., Smith, S.: Segmentation of brain MR images through a hidden Markov random field model and the expectation-maximization algorithm.IEEE trans. on medical imaging 20 (2001) 45

[14] Digital Image Processing, by Rafael C. Gonzalez, Richard Eugene Woods.

[15] Digital Image Processing, by Kenneth R. Castleman

[16] Digital Image Processing Using Matlab, by Rafael C.Gonzalez, Richard E. Woods, Steven L. Eddins

[17] Fundamentals of Digital Image Processing, by Anil K. Jain

[18] Digital Image Processing, by Bernd Jähne

[19] Digital Image Processing Algorithms and Applications, by Ioannis Pitas

[20] Graphics and GUIs with MATLAB: 2e. Patrick Marchand. CRC Press, Inc., 1999 (ISBN 0-84939487-2). 